\title{
Entwicklungstendenzen des finnisch-ugrischen Kasussystems ${ }^{1}$
}

Die finnisch-ugrischen Sprachen sind sich ihrer morphologischen Struktur nach ziemlich ähnlich. Ein Teil der Übereinstimmungen kann direkt aus der finnisch-ugrischen oder sogar uralischen Ursprache hergeleitet werden, während ein anderer Teil das Ergebnis einer späteren gleichlaufenden Entwicklung ist. Gerade die letzterwähnten, auf Konvergenz hinweisenden Fälle sind ein vom allgemeinen Standpunkt sehr interessantes, vorläufig aber wenig erforschtes Gebiet. Zeigen sie doch, sofern es sich nicht um einen späteren gegenseitigen oder durch irgendeine nichturalische Sprache verursachten Lehn- oder Substrateinfluss handelt, dass es in der fiu. Grundsprache Strukturmerkmale gegeben hat, die die Entwicklung der Tochtersprachen noch Jahrtausende nach der Aufspaltung der Ursprache lenken. Dass in einer Sprache solche langfristigen Entwicklungstendenzen auftreten, weiss man schon lange (vgl. die "Tendenz" bei Meillet und den "drift" bei Sapir), doch wissen wir noch recht wenig darüber, worauf sie beruhen und wie die dafür zuständigen Mechanismen in einer jeden Sprache genau genommen arbeiten. Ich möchte im folgenden Übereinstimmungen in der Entwicklung der Kasussysteme der fiu. Sprachen im Hinblick darauf untersuchen, inwieweit sie von den Strukturmerkmalen der Grundsprache ableitbar sind.

${ }^{1}$ Dieser Aufsatz basiert auf einem Vortrag, den ich am 17. 2. 1978 anlässlich der Sprachwissenschaftlichen Tagung in Joensuu hielt. Ich danke Herrn Doz. Esa Itkonen für seine Stellungnahme zu meinem Vortrag. 
1. Die Anzahl der Kasus in den finnisch-ugrischen Sprachen

Der Kasusreichtum ist für die meisten finnisch-ugrischen Sprachen eine typische Erscheinung. Das Finnische verfügt über 15 Kasus, das Mordwinische über 9-11 und das Tscheremissische über 7-13, abhängig davon, was man als Kasus betrachtet, das Syrjänische besitzt 17, das Wotjakische 16 und das Ungarische 17-23. Eine Ausnahme bilden das Lappische und die obugrischen Sprachen, die weniger Kasus haben, das Lappische - abhängig vom Dialekt - immerhin 7-9, das Wogulische 6-7 und das Ostjakische dialektweise nur 3.

Für die fiu. und auch die ural. Grundsprache werden allgemein sechs Kasus angenommen: ein endungsloser Nominativ, ein $n$-Genitiv, ein $m$-Akkusativ sowie die Lokalkasus: ein Lativ mit den Endungen $n$ und $k$, ein Lokativ mit der Endung $n a / n \ddot{a}$ und ein Ablativ mit der Endung ta/tä. Diese Rekonstruktion ist natürlich nur durch die vergleichende Methode zustande gekommen und kann somit nicht als unbedingt sicher gelten. Es ist jedoch die wahrscheinlichste Rekonstruktion, von welchem Standpunkt man die Dinge auch betrachtet. Was die allgemeine Struktur des Kasussystems und die Anzahl der Kasus angeht, weichen die in der letzten Zeit vorgebrachten Rekonstruktionen nicht nennenswert vom Obigen ab. (Vgl. Szinnyei 1922 S. 55-63, Collinder 1960 S. 238, E. Itkonen 1962 S. 201-202, Osnovy 1974 S. 232, Hajdú 1976 S. 62.)

Noch heute besteht der Rahmen des Kasussystems in den meisten fiu. Sprachen aus Nominativ, Genitiv, Akkusativ sowie einer dreiteiligen Lokalkasus-Serie, wozu eine wechselnde Anzahl Wohin-, Wo- und Woher-Kasus gehören. Dieselbe Situation gilt für die samojedischen Sprachen. Zu ihrem Kasusparadigma gehören jene drei sog. grammatischen Kasus, Nominativ, Genitiv und Akkusativ, sowie drei Lokalkasus, Lativ, Lokativ und Ablativ; zusätzlich können 1-3 lokale oder komitativ-instrumentale Kasus (Prolativ, Translativ, Instrumental) auftreten. Die Rekonstruktion des Kasussystems der uralischen Ursprache beruht wesentlich gerade auf jener für die heutigen ural. Sprachen typischen Struktur des Kasussystems.

Nach der fiu. Zeit hat die Anzahl der Kasus in den meisten 
Sprachen zugenommen. Die Erweiterung betraf vor allem das Teilsystem der Lokalkasus, das sich in den meisten Sprachen verdoppelt oder verdreifacht hat. Auch ausserhalb des eigentlichen Lativ-, Lokativ- und Ablativsystems sind neue Lokalkasus entstanden, wie Prolativ, Transitiv, Terminativ und Prosekutiv. Unter den neuen Kasus finden sich auch häufig komitativ-instrumentale Kasus, in deren semantisches Feld auch der Karitiv und der Abessiv gehören. In geringerem Umfang hat sich das Wachstum auf andere Adverbialkasus bezogen.

Der Kasusreichtum der fiu. Sprachen geht also nicht als solcher auf die fiu. Ursprache zurück, sondern ist eine Folge der später in einer jeden Sprache oder Sprachgruppe vollzogenen Entwicklung. Da sich jedoch bei den meisten dieser Sprachen eine Zunahme der Kasus feststellen lässt, liegt der Gedanke nahe, dass die fiu. Ursprache über strukturelle Merkmale verfügte, durch die die Entwicklung der Tochtersprachen für Jahrtausende vorprogrammiert wurde.

Natürlich sind die Kasussysteme, wie sie hier geschildert werden, in keiner Weise autonome morphologische Teilsysteme; im Gegenteil: sie sind ganz stark an die Syntax und Phonologie der Sprache gebunden. So sucht man die Wurzeln der von uns untersuchten Erscheinung sicher vergeblich nur in den inneren Eigenschaften des Kasussystems. Eher muss die Aufmerksamkeit jenen syntaktischen Beziehungen gelten, denen das Kasussystem dient und die somit auf die Entwicklung des Kasussystems einwirken, sowie jenen Grundzügen der phonologischen Struktur, durch die die Morphophonemik der Sprache und damit auch der Ausdruck der syntaktischen Beziehungen gelenkt wird.

\section{Die Bildungsprinzipien neuer Kasus}

Die Entwicklungsprozesse, die in den finnisch-ugrischen Sprachen nach der Zeit des Zusammenlebens zu neuen Kasus geführt haben, sind in etwa bekannt. In der Hauptsache sind die neuen Kasus auf zwei alternativen Wegen entstanden, entweder aus Postpositionskonstruktionen oder durch neue Suffixkombinationen. Im folgenden wollen wir uns diese Prozesse näher ansehen. 
2.1. Aus einer Postpositionskonstruktion kann ein Kasus entstehen, wenn die Verbindung von Bezugswort und Postposition so eng wird, dass sie als ein Wort aufgefasst wird, wo der die Postposition vertretende Teil zu einem lediglich formalen Element und gleichzeitig lautlich vereinfacht wurde. Bei dem Prozess kommt es generell zu sowohl semantischen als auch morphophonemischen Veränderungen, die die Wortgrenze verschwinden lassen und den Status der Teile verändern.

Kasus, die aus Postpositionskonstruktionen entstanden sind, begegnen vor allem in den ugrischen Sprachen, im Ungarischen, Ostjakischen und Wogulischen. Wir brauchen die Kasusbildung in den ugrischen Sprachen hier nicht detailliert zu schildern, denn sie ist grundsätzlich geklärt (s. z.B. Szinnyei 1927 S. 129-137, Bárczi - Benkő - Berrár 1967 S. 403-409, Liimola 1963 S. 24-201). Z.B. von den Kasus des Ungarischen sind bekanntlich ca. ein Dutzend ziemlich spät aus Konstruktionen mit Postpositionen entstanden. Um den Prozess zu veranschaulichen, wollen wir ein bekanntes Beispiel heranziehen, die inneren Lokalkasus des Ungarischen. Ihre Endungen sind aus alten Lokalkasusformen des Substantivs bél 'Innen-, Inneres; Darm, Eingeweide; Kern' entstanden, die als Postpositionen verwendet wurden, z.B.

Iness. Sg. kéz-ben 'in der Hand' < -beln < *belV-n< *pälV-nä (*käte pälVnä 'im Innern der Hand');

Ill. Sg. kéz-be 'in die Hand' <-bele <-belé < *belV-i< *päl $\mathrm{V}-k / j$;

Elat. Sg. kéz-böl 'aus der Hand' < -belöl < *bele-ül < $?^{*} p a ̈ l \mathrm{~V}-l(\mathrm{~V})$.

Durch die Fusion der Postposition mit ihrem Bezugswort kam es sowohl zu semantischen als auch zu morphophonemischen Veränderungen. Die Bedeutung mutierte vom konkreten 'Innern' zum Ausdruck eines bestimmten adverbialen Verhältnisses. Die äusseren Kennzeichen der Fusion sind Vokalharmonie, z.B. kézben 'in der Hand' házban 'im Haus', und Stimmtonassimilation, z.B. kertben [kerdben] 'im Garten' $(t>$ $d$ vor einem stimmhaften Konsonanten). Gerade für das Ungarische ist jedoch festzustellen, dass die Vokalharmonie nicht die Bedingung dafür ist, dass eine Konstruktion als ein Wort 
verstanden wird. Von den Kasusmorphemen des Ungarischen steht eins deutlich ausserhalb der Vokalharmonie, nämlich -kor, die Endung des Temporals, z.B. hatkor 'sechs Uhr' $\sim$ ötkor 'fünf Uhr'. Ausserdem gibt es einige Endungen mit dem neutralen Vokal $e$ oder é, deren Phonotaktik nicht unbedingt ein hintervokalisches Allomorph verlangt, z.B. den Essiv-Formal (fal-ként 'als Mauer'), den Kausal-Final (fal-ért 'wegen der Mauer') und den Formal (példa-képpen 'zum Beispiel'). Diese Kasusendungen weisen jedoch eine um einen Grad lockerere Fusion auf als die ebenfalls ein $e$ oder é enthaltenden Morpheme, wie z.B. die Endungen von Inessiv und Illativ, die sich der Vokalharmonie angepasst haben.

Obgleich das Kasussystem des Wogulischen als Ganzes dem angenommenen fiu. System nahesteht, gehen die morphologischen Elemete seiner einzelnen Kasus durchaus nicht alle auf die Ursprache zurück. Nach Liimola (1963) sind wenigstens der Elativ-Ablativ (-nəl), der Lativ (-nà, -nä̀ usw.) und in den Süddialekten der Komitativ (-nàt, -nàt) im eigentlichen Substantivparadigma aus Postpositionskonstruktionen entstanden.

Die drei Grundkasus der Kasussysteme in den ostjakischen Dialekten, Nominativ, Lokativ und Lativ, gehen alle auf die uralische Grundsprache zurück. Zusätzlich dazu begegnen dialektal auch andere Kasus, vor allem aus Konstruktionen mit Postpositionen entstanden, wie der Komitativ (-nât) und der Lativ (-nà).

Von den Kasusendungen postpositioneller Herkunft in den ugrischen Sprachen ist ein Teil schon in urugrischer Zeit entstanden. Hierher scheinen wenigstens die folgenden $\mathrm{zu}$ gehören, die aus den Lokalkasus einer $*_{n} \mathrm{~V}$-stämmigen Postposition entstanden sind: der Komitativ-Lokativ (vgl. wogS Kom. ämpnä̀t 'mit dem Hund', ostjO Kom. iminàt 'mit der Frau', ungN O Lok. bírónott, bírónitt 'beim Richter, in der Familie des Richters'), der Elativ-Ablativ (vgl. wogS külnà̀ $\cdot l$ 'aus dem Haus', ungN O bírónól, -núl, -nul 'vom Richter, aus der Familie des Richters') und der Lativ (vgl. wogS ōšna. 'in die Stadt', ostjN s ̇̀mnà 'vor die Augen', ungN O bíróni, -nyi 'dem Richter, zur Familie des Richters'). Diese Kasusendungen sind allerdings auch anders ausgelegt worden, dass sie nämlich so 
entstanden wären, dass die Endungen des Lokativs $\left({ }^{*} t t(\mathrm{~V})\right)$, des Ablativs (*-l) und des Lativs (*-k) an die uralische Lokativendung *na/nä getreten wären; aufgrund der letzten Untersuchungen, vor allem der gründlichen Ausführungen von Liimola, scheint jedoch die von Postpositionskonstruktionen ausgehende Erklärung glaubwürdiger zu sein (Liimola 1963 S. 64-73, 92-98, 120-123).

Von den sekundären Kasus des Ungarischen ist jedoch der grösste Teil in der Zeit der ungarischen Sonderentwicklung, teilweise erst in historischer Zeit, entstanden und der Struktur nach noch sehr durchsichtig.

Im finnisch-permischen Zweig hat man viel weniger Kasusendungen nachweisen können, die aus Postpositionen entstanden sind. Aus den ostseefinnischen Sprachen sind folgende durch recht späte Fusion entstandene Kasus bekannt: der $-k a$ $(-k a ̈)$ (< kanssa 'mit') -Komitativ, der im Livischen, Estnischen, Wotischen, in den ingrischen Dialekten und dialektweise im Finnischen begegnet, ein -ke (<kera, keralla) -Komitativ, der im Karelisch-Olonetzischen, in den lüdischen Mundarten und im Wepsischen auftritt, im Wepsischen ein -mö, -mo usw. (< *mötä) -Prolativ-Komitativ, im Karel.-Olon., Lüd. und Weps. -luo, -lo usw. -Peripherale, wozu ein Wohin-, Wo- und WoherKasus gehören kann, sowie im Wot., in den ingrischen Dialekten, im Olon., in den lüdischen Dialekten und im Weps. ein -sa, -sā usw. (< * sānnik, *sākka usw.) -Terminativ (vgl. Oinas 1961). In all diesen Fällen ist die Fusion so jung, dass sich die entsprechende Postpositionskonstruktion noch neben der Suffixkonstruktion erhalten hat. Auch die zur Postpositionskonstruktion gehörende Genitiv- oder Partitivform des Bezugswortes ist meist noch vor dem Suffix zu erkennen. Die Konstruktionen haben noch nicht in allen Fällen ganz unumstritten den Status eines Kasus.

Recht transparent ist auch der Komitativ Pl. im Lappischen, dessen Endung -guim (z.B. lpN nieidâiguim 'mit den Mädchen') sich aus der Postposition guoime entwickelt hat. Diese wiederum ist eigentlich der instrumental verwendete Gen.Sg. vom Wort guoi'bme 'Kumpel, Genosse, Ehepartner' ( fi. kaima < balt.) (SKES 1955 s.v. kaima). 
Aus dem Mordwinischen seien zwei Kasus erwähnt, die aus einer Konstruktion mit Postposition entstanden sind und im Mokscha vorkommen: ein Dativ (z.B. v́el'ańd'i 'dem Dorf'), dessen Endung -ńd'i $<*_{-}^{*} n$ (Gen.) + ${ }^{*} t e j \mathrm{~V} j$ (Lativ des Wortes t'ej $\sim$ fi. tyvi), und ein Kausativ (z.B. modanksa 'wegen der Erde'), dessen Endung - $\eta k s a<$ i $k$ ksa (Postp.) 'wegen, um' (Posti 1937 S. 289, Osnovy 1975 S. 292).

Das elativische Morphem des Tscheremissischen kats(an),

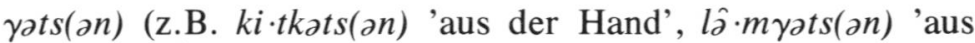
dem Schnee'), das in den früheren grammatischen Darstellungen oft als Kasusendung betrachtet wurde, ist nach der neueren Deutung auch heute noch eine Postposition (Alhoniemi 1968 a S. 71, 1968 b S. 7, 1977 S. 5).

Im Wotjakischen, zumindest in den Mundarten von Glazov und Besserman, begegnen der sog. Adessiv II (z.B. mumez-ńin 'bei ihrer Mutter') und der Allativ II (z.B. mumiz-ń $\varepsilon$ 'zu ihrer Mutter'), deren Endungen aus den Postpositionen dińîn 'bei' und diń $\varepsilon$ 'zu' entwickelt sind, die wiederum ursprünglich Inessiv- und Illativformen des Wortes din 'dickes Ende eines Baumstammes' darstellen: -ńîn < dińîn, -ń $\varepsilon<$ diń $\varepsilon$ (Wichmann-Fuchs 1954 S. 135-136). Im permjakischen Dialekt des Syrjänischen haben sich wiederum die Postpositionen vilin 'auf (Dat.)', viliś 'von oben weg', vile 'auf (Akk.)', und vilet' 'oben drüber' zu den Kasusendungen -vin, -vvin, -vviś, -ve, -vve, -vett', -vvet $t^{\prime}$ entwickelt (z.B. ib-vin 'auf dem Felde', ib-ve 'auf das Feld', ib-vet t' 'auf dem Felde') (Wichmann-Uotila 1942 S. 404, Rédei 1962 S. 207).

Die Komitativendung -mid (z.B. piidmid 'mit deinem Sohn') im Dialekt von Luza des Syrjänischen könnte nach Wichmann (1924 S. 152) auf eine Postposition zurückgehen: mi- 'hinten' ( ung. mög, fi. myö- usw.) $+-d$ (Prosekutiv-Endung), welche Erklärung jedoch durch Fuchs überzeugend widerlegt worden ist (1927 S. 198-205). Auf noch unsicherem Boden stehen die Versuche, die syrj. Komitativ-Endung -ked (z.B. mortked 'mit dem Menschen' und die tscher. Komitativ-Endung -ke, - $\gamma e$

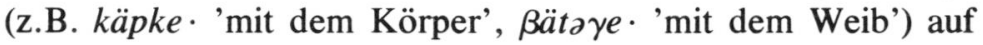
Postpositionen zurückzuführen (Budenz 1873-81, S. 65-66, Paasonen 1897 S. 15-16, Wichmann 1924 S. 153-154). 
Aus dem finnisch-permischen Zweig lassen sich also endgültig nur wenige sichere Kasusendungen postpositioneller Herkunft nachweisen. Auch von ihnen sind die meisten so jung, dass sich die ursprüngliche Postpositionskonstruktion noch neben dem Kasus erhalten hat.

Die Fusion von Postpositionskonstruktionen und die dadurch eintretende Neugliederung in der Grammatik zu einem Teil des Kasussystems erklärt sich im Prinzip recht geradlinig aus zwei Grundeigenschaften der Struktur der fiu. Ursprache und auch der meisten heutigen fiu. Sprachen:

1. Der Modifikator steht vor dem Bezugswort. Bekanntlich ist die Postposition ursprünglich das Bezugswort gewesen und das heutige Bezugswort war ihr Attribut. Noch heute lässt sich z.B. eine solche fi. Konstruktion wie kiven vieressä 'neben dem Stein' leicht entweder so verstehen, dass kiven das Bezugswort und vieressä die dazugehörige Postposition darstellt, oder so, dass kiven ein Genitivattribut für die Inessivform des Wortes vieri ausmacht.

2. Der Hauptakzent liegt auf der ersten Silbe des Wortes oder auch der Phrase. In einer Konstruktion mit Postposition fällt der Hauptakzent auf die erste Silbe des Bezugswortes, während die erste Silbe der Postposition nur einen Nebenakzent erhält oder völlig unbetont bleibt. Damit gerät die Postposition in eine suffixartige Stellung und die ursprüngliche Postpositionskonstruktion wird phonotaktisch und morphophonemisch als ein Wort behandelt. Einen Einfluss haben darauf z.B. die Regeln über die Kombinierung der Konsonanten und über die Vokalharmonie. Die an der Fusion beteiligte Postposition gerät aber ausserdem ihrer Unbetontheit wegen in die für unbetonte Silben im letzten Teil des Wortes typische vereinfachende Lautentwicklung, wie Schwächung oder Schwund des Konsonantismus, Apokope oder Silbenschwund. Als morphologisches Element ist sie ausserdem der Analogie ausgesetzt.

Wir können also feststellen, dass die Grundwortstellung (Modifikator vor Bezugswort) die Entstehung des Postpositionssystems ermöglicht und das Akzentsystem (Hauptakzent auf der ersten Silbe) die Fusion der Postpositionen zu suffixartigen Elementen erleichtert hat. Der auf die erste Silbe fallende 
Hauptakzent ist jedoch keine unumgängliche Bedingung, wie die späten Fusionsfälle im Wotjakischen zeigen; im Wotjakischen liegt ja der Hauptakzent auf der letzten Silbe des Wortes.

Alle oben angeführten Fusionsfälle mit Ausnahme der urugrischen Kasusendungen, die aus $*_{n} \mathrm{~V}+\mathrm{Kasusendung}$-Postpositionen entstanden sind, haben kein sehr hohes Alter, weshalb die durch Fusion entstandenen Kasusformen ihrem Aufbau nach recht durchsichtig sind. Möglicherweise hat es eine entsprechende Entwicklung auch in den früheren Entwicklungsphasen der fiu. Sprachen gegeben, da aber gerade die suffixalen Silben recht anfällig sind gegenüber Abnutzung und Analogieveränderungen, sind die Spuren sehr alter potentieller Fusionen verschwunden. Deshalb könnte z.B. eine solche Annahme, dass die finnisch-permischen $l$-Kasus (u.a. die äusseren Lokalkasus des Finnischen, z.B. kadulla, kadulta, kadulle) und die finnisch-wolgaischen $s$-Lokalkasus (u.a. die inneren Lokalkasus des Finnischen, z.B. talossa, talosta, taloon) durch Fusion von luo- und sisä-stämmigen Postpositionen mit dem Bezugswort entstanden wären (z.B. in der Art: *pesän lōna > *pesänlenä $>$ *pesälenä $>{ }^{*}$ pesälnä $>$ pesällä; *pesän sisänä $>$ *pesänsisnä > *pesänsnä > *pesäsnä > pesässä) nicht als richtig bewiesen werden, wenn man sie auch schwerlich bindend widerlegen könnte. An sich läge hier eine folgerichtige Realisierung jener Entwicklungslinie vor, die wir aus der Kasusgeschichte der fiu. Sprachen aufgrund der obigen Ausführungen als recht allgemein erkannt haben. In Wirklichkeit ist jedoch für die Entstehung dieser Kasus eine ganz andere, viel glaubwürdigere Erklärung vorgebracht worden, ausgehend von dem zweiten Hauptmechanismus, der für die Entstehung neuer Kasus in den fiu. Sprachen zuständig war.

2.2. Der zweite zur Erweiterung der Kasusanzahl führende Prozess, die Produktion neuer Kasusmorpheme durch Verbindung von einfachen Suffixen zu Suffixkomplexen, war in der finnisch-permischen Gruppe besonders beliebt, während der ugrische Zweig weniger Anzeichen davon aufweist. Die älteste diesbezügliche Schicht dürften die finnisch-permischen lokalhabitiven Kasus mit $l$ vertreten: die sog. äusseren Lokalkasus des Osfi., der Adessiv (z.B. isä-llä), der Ablativ (isä-ltä) und 
der Allativ (isä-lle), im Tscher. der Allativ-Dativ ( $k i \cdot$ Dlän 'zur Hand, als Hand'), der Ablativ ( $k i \cdot D$-letš 'von der Hand') und der Modal ( $k i \cdot D$-lä 'wie die Hand') sowie die habitiven Kasus der perm. Sprachen, der Adessiv bzw. Genitiv (z.B. syrj. mort-len 'des Menschen, beim Menschen'), der Allativ bzw. Dativ (syrj. mort-li 'dem Menschen') und der Ablativ (syrj. mort-liś 'vom Menschen') sowie der Approximativ (syrj. mort-lań 'in Richtung auf den Menschen') und der Konsekutiv (syrj. pu-la 'nach Holz, Holz holen'). Allerdings hat das Fehlen dieses Kasustyps im eigentlichen Substantivparadigma des Lappischen und Mordwinischen Anlass zu der Annahme gegeben, dass er auch in der finnisch-permischen Ursprache noch nicht zum System der eigentlichen Kasus gehört sondern eher eine produktive Partikel-Ableitungsgruppe gebildet habe. Jedenfalls denkt man sich seine Entstehung so, dass an die mit dem lokalen Suffix $l$ gebildeten Partikeln die primären Lokalkasusendungen traten, z.B. im Osfi. ${ }^{*} l+n a / n a ̈,{ }^{*} l+$ taltä und ${ }^{*} l+n$. (Szinnyei 1922 S. 63-65, Ravila 1935 S. 43-45, Hakulinen 1968 S. 90-91.)

Aus der finnisch-wolgaischen Epoche stammen die Lokalkasus mit $s$ : im Osfi. die inneren Lokalkasus, der Inessiv (pesässä), der Elativ (pesä-stä) und der Illativ (pesä-än < *pesäsen), im Lappischen der Inessiv (lpS goåt'a-sn 'im Zelt'), der Elativ (goåt'ə-st'ə 'aus dem Zelt') und Illativ (becerg as a 'ins Fleisch'), im Mordwinischen der Inessiv (moda-s̀o 'in der Erde'), der Elativ (moda-sto 'aus der Erde') und der nur in Partikeln begegnende Illativ (ko-zoń 'wohin') sowie im Tsche-

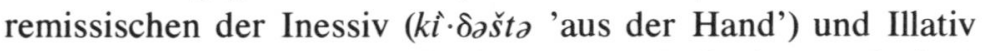
$(k \hat{i} \cdot \delta \partial s ̌ k \partial$ 'in die Hand'). Hier ist an den als finnisch-wolgaisch anzusehenden $s$-Lativ (z.B. fi. ulos 'hinaus', alas 'hinunter') eine primäre Lokalkasusendung getreten. Dass gerade der Lativ den Rahmen für das neue System übernahm, nimmt nicht wunder, denn wie ich an anderer Stelle (1975) nachzuweisen versuchte, war gerade der Wohin-Kasus die kennzeichenlose Grundkategorie des fiu. Lokalkasussystems.

Auch der Translativ ist zumindest finnisch-mordwinischer Herkunft, vgl. fi. maaksi 'zu Erde', lp. Partikel luk'sâ 'nach Süden', mordw. modaks 'zu Erde'. Hier sind zwei Lativendun- 
gen vereint: $*_{-} k$ und $*_{-s}(e)$.

Ein offensichtlich altes, vielleicht schon auf die finnisch-permische Zeit zurückgehendes zusammengesetztes Suffix ist das abessiv-karitive $*$-ptalptä- $+-k$ (nach einigen Auffassungen *-ttalttä- + -k, z.B. fi. raha-tta 'ohne Geld', leivä-ttä 'ohne Brot', lpN gieđâ-tâgâ 'ohne Hand', S mecesakâ-pth 'ohne Fell/Haut des Rentierkalbs', tscher. ki-t-tè 'ohne Hand', syrj. kerka-teg 'ohne Haus', wotj. mumi-tek 'ohne Mutter' (s. Korhonen 1974 S. 173-176 und die dort genannte Literatur).

Ausser den oben behandelten finden wir in den finnisch-permischen Sprachen noch isolierte jüngere Kasus, die eine geringere Verbreitung haben und mit einer zusammengesetzten Endung versehen sind, wie den osfi.-lapp. Komitativ mit der Endung $*_{-j-}+-n e$ (oder -na/nä) $(+-k)$ (z.B. fi. pitk-ine jalko-ine-en 'mit seinen langen Beinen', $\mathrm{lpN}$ gieđâ-in 'mit der Hand', vgl. auch mordw. pargo-ńek 'mit seinem Korb'), in den perm. Sprachen den Transitiv mit der Endung - $t i,-t i,-k i(<-t t \mathrm{~V}$ $+-j$ ) (z.B. syrj. bereg-t $i$ 'das Ufer entlang', wotj. tele-ti 'durch den Wald'), im Syrjänischen den Präklusiv auf -śa (z.B. mort-śa 'ausser dem Menschen'), den im Syrj. auf -śań und im Wotj. auf -śen ausgehenden Egressiv (z.B. syrj. mort-śań 'vom Menschen weg', wotj. korka-śen 'aus dem Haus, vom Hause'), von denen die drei letztgenannten ausser dem für die permischen Sprachen charakteristischen elativischen ś-Element noch andere Suffixelemente enthalten $(-a,-n,-n)$ (Ravila 1941 S. 44-46, Rédei 1968 S. 171-173, Uotila 1933 S. 314, E. Itkonen 1966 b S. 274-275).

Der vielleicht einzige in diese Gruppe gehörende Kasus in den ugrischen Sprachen ist der ungarische Komitativ, z.B. családo-stul 'mit seiner Familie' (<*-s- (Adjektivsuffix) $+-t$ (Lok.) + -ul (Ess.) (Klemm 1928 S. 214, Ravila 1941 S. 44).

Obgleich die Entstehung der Kasus durch Suffixkombinationen in fast jedem bekannten Fall zufriedenstellend beschrieben werden kann, ist es doch nicht leicht, für diese Fälle eine solche gemeinsame Erklärung zu finden, wie es bei den Postpositionskonstruktionen möglich war. Wenn wir davon ausgehen, dass das Kasussystem in den uralischen Sprachen stets durch das Postpositionssystem ergänzt wurde, wie in den indoeuropäi- 
schen Sprachen durch das Präpositionssystem, so stellt sich die Frage, warum das nicht ausreichte, sondern warum man ausserdem zusätzlich noch ganze Kasusreihen brauchte, deren Endungen eben aus Suffixkombinationen bestanden. Eine erschöpfende Antwort kann auf diese Frage schwer gegeben werden; man kann jedoch auf einige Zusammenhänge hinweisen.

Zunächst muss daran erinnert werden, dass die oben erwähnte Wortfolgeregel der uralischen Sprachen "'Modifikator vor Bezugswort" direkt zur Entstehung des Postpositionssystems und zur Erweiterung des auf Postpositionen aufbauenden Kasussystems geführt hat, d.h. zu einem System, wo die syntaktischen Verhältnisse durch grammatische Morpheme hinter dem Stamm ausgedrückt werden. Dadurch wurde wiederum die Entwicklung eines Präpositionssystems und der damit leicht einhergehende Verfall des Kasussystems verhindert. In den indoeuropäischen Sprachen z.B. hatte das Präpositionssystem ursprünglich nur das Kasussystem zu ergänzen, doch erhält in der linearen Morphemkette die zuerst stehende Präposition eine im Informationswert wichtigere Position als das erst später stehende Kasusmorphem, wodurch die Erhöhung der Redundanz der Kasusmorpheme die Grundlage bildet für den Verfall des Kasussystems. Bereits dieser Unterschied zwischen Präpositions- und Postpositionssprachen schafft meines Erachtens die Voraussetzungen für eine unterschiedliche Entwicklung des Kasussystems in diesen Sprachtypen. Offenbar führt die Wortfolge "'Modifikator vor Bezugswort" nicht nur zum Postpositionssystem und zu einer auf Postpositionen basierenden Erweiterung des Kasussystems, sondern es bietet auch die Grundlage für eine andersgeartete Entwicklung eines Suffixsystems zur Erzeugung neuer Kasus.

Ein zweiter Faktor, der auf die Entstehung zusammengesetzter Kasusendungen eingewirkt hat, ist eine Erscheinung, die Charles S. Peirce als Ikonizität bezeichnet hat (1955 S. 98-119). Er teilt die Zeichen in drei Grundtypen: Ikone, Indexe und Symbole. Das Ikon zeigt die formale Ähnlichkeit zwischen dem Zeichen und seiner Referenz. In der Morphologie kommt die Ikonizität als eine Kraft zum Ausdruck, die die gegenseitige 
Entsprechung zwischen Form und Bedeutung möglichst vollständig beibehalten will. Die Formstruktur ist gleichsam die Abbildung der Bedeutungsstruktur. Diese Art der Ikonizität heisst Diagrammhaftigkeit. Das Diagramm ist das Ikon der Verhältnisse. Der von Peirce entwickelte Begriffsapparat ist u.a. von Roman Jakobson (1965) und Raimo Anttila (u.a. 1972, 1977) bekannt gemacht worden, und gerade der Begriff der Ikonizität hat sich in der letzten Zeit speziell in der Morphologie als fruchtbar erwiesen. Er scheint auf einer festen, psychologisch motivierbaren Basis zu stehen und bringt auch für die Analogie ein allgemeines Erklärungsprinzip: jener Änderungsprozess, der die Ikonizität in der Sprache verwirklicht, ist oft gerade die Analogie (s. Anttila 1977).

Wie hat nun die Ikonizität die Entstehung der zusammengesetzten Kasusendungen in den fiu. Sprachen beeinflusst? Betrachten wir zunächst die frühurfinnischen Rekonstruktionen der allgemeinen, inneren und äusseren Lokalkasus des Finnischen im Lichte des folgenden Beispiels:

\begin{tabular}{|c|c|c|c|}
\hline & allgemeine $\mathrm{L}$. & innere L. & äussere L. \\
\hline Wohin? & ${ }^{*}$ pesä-k/n/s & *pesä-se-n & *pesä-le-n \\
\hline Wo? & *pesä-nä & *pesä-s-nä & *pesä-l-nä \\
\hline Woher? & *pesä-tä & ${ }^{*}$ pes $\ddot{a}-s-t \ddot{a}$ & *pesä-l-tä \\
\hline
\end{tabular}

(vom Wort pesä 'Nest').

Dieses Beispiel, das ein Teilsystem des frühurfinnischen Kasussystems veranschaulicht, bringt die Ikonizität sehr stark zum Ausdruck. Wir sehen darin wenigstens zweierlei systematische Diagrammhaftigkeit. Zunächst besteht das Bestreben, die Begriffe 'Wohin?', 'Wo?' und 'Woher?' jeweils mit einem eigenen Zeichen auszudrücken. Das Zeichen des 'Wohin?' ist -n, das des 'Wo?' -na/nä und das des 'Woher?' -ta/tä. Zweitens unterscheiden wir das Zeichen des Inneren -s(e)- und das des Äusseren -l(e)-. In diesen Diagrammen erhalten die Teilsysteme des Kasussystems, wie die inneren Lokalkasus, die habitiv-lokalen Kasus, die Wohin-, Wo- und Woher-Kasus ein eindeutiges morphologisches Kennzeichen. 
Auffallend ist, dass die Ikonizität im ursprünglichen fiu. Kasussystem nicht verwirklicht wird. Diese Tatsache lässt sich durch folgende Rekonstruktion veranschaulichen:

\begin{tabular}{lllll}
\hline fiu. & Nom. & ${ }^{*}$ pesä & Lat. & ${ }^{*}$ pesä- $k / n$ \\
& Gen. & ${ }^{*}$ pesä- $n$ & Lok. & ${ }^{*}$ pesä-nä \\
Akk. & ${ }^{*}$ pesä- $m$ & Abl. & ${ }^{*}$ pesä-tä \\
\hline
\end{tabular}

Wir sehen, dass das Lokalkasussystem durch kein Diagramm als Teilsystem des gesamten Kasussystems gekennzeichnet ist. Die Entstehung neuer Kasus bedeutete also eine Zunahme der Ikonizität auf dem Wege von der fiu. Ursprache zum Frühurfinnischen. Dasselbe Merkmal lässt sich auch in anderen fiu. Sprachen feststellen, bei denen die Anzahl der Mitglieder des Kasussystems gewachsen ist.

Die Ikonizität verwirklicht sich wie gesagt vor allem durch Analogie. Ein gutes Beispiel hierfür ist die Geschichte der finnisch-wolgaischen $s$-Kasus: Zunächst gab es den $s$-Lativ (z.B. *pesä-s). Damit wurden analog die Endungen der alten Lokalkasus verbunden, Lok. *-na/nä und Abl. *-taltä. Auf diese Weise erhielt man einen Inessiv (*pesä-s-nä) und einen Elativ (*pesä-s-tä). Später - erst im Frühurfi. - wurde dann unter Verwendung des Inessiv- und Elativmodells analog der Illativ (*pesä-se-n) gebildet, indem das alte Lativ-* $n$ mit dem als Sekundärstamm aufgefassten $s$-Lativ verbunden wurde. (Korhonen 1975.)

Die Ikonizität an sich ist jedoch eine universale Tendenz. Sie allein kann also nicht die Erweiterung des Kasussystems durch Suffixkombinationen verursachen. Als Ausdruck der Ikonizität kann ebensogut z.B. das Präpositionssystem des Deutschen angesehen werden. Damit die Ikonizität ihren Niederschlag in Suffixkombinationen findet, muss auch die zweite Bedingung erfüllt sein, nämlich die Wortfolge '"Modifikator vor Bezugswort', die - wie oben festgestellt - suffixale Kasus begünstigt. So kommen wir zu dem Schluss, dass sowohl die auf Postpositionen basierende als auch die durch Suffixkombinierung erfolgende Erweiterung des Kasussystems in den fiu. Sprachen letzten Endes auf dem Kasussystem und der Grundwortfolge 
beruhen, die bereits in der fiu. Grundsprache herrschend waren.

\section{Postpositionen oder Suffixkombinationen?}

Woher kommt es nun, dass die Erweiterung des Kasussystems in den ugrischen Sprachen hauptsächlich auf der Postpositionstechnik, in den finnisch-permischen Sprachen aber auf der Suffigierungstechnik beruht? Handelt es sich hier auch um Strukturunterschiede der ugrischen und finnisch-permischen Grundsprache? Hätten die möglicherweise verschiedenen Grundstrukturen dieser Sprachen als Prädispositionen für die Entwicklung gewirkt?

Auf den ersten Blick ist nur ein Unterschied zu erkennen, der hier von Bedeutung sein könnte: in den ugrischen Sprachen treten die Postpositionen immer an den Nominativ, in den finnisch-permischen dagegen teilweise an den Nominativ, teilweise an den Genitiv, mitunter auch an andere Kasus. Der uralische $n$-Genitiv hat keinen Vertreter in den ugrischen Sprachen, so dass er schon im Urugrischen geschwunden sein dürfte. Da auch in den samojedischen Sprachen das Bezugswort der Postposition allgemein im Genitiv steht, hat offenbar schon in der uralischen Grundsprache ein System geherrscht, wo die Postpositionen mit dem Genitiv und möglicherweise mit dem Nominativ verbunden wurden, was auch ganz natürlich ist, wenn wir die Herkunft der Postpositionskonstruktionen berücksichtigen. Das ururalische Postpositionssystem dürfte als solches in die finnisch-ugrische Ursprache übernommen worden sein. Der Schwund des Genitivs im Urugrischen bedeutete unbedingt auch eine Veränderung des Postpositionssystems in diesem Zweig der finnisch-ugrischen Sprachen: die Postpositionen wurden danach nur mit dem Nominativ verbunden.

Auch in den permischen Sprachen ist der $n$-Genitiv geschwunden, obgleich er in seiner komitativ-instruktiven Funktion als Instrumentalkasus weiterlebt. Die Aufgaben des Genitivs sind teilweise auf den zu den $l$-Kasus gehörenden Adessiv mit der Endung -lęn, -len übergegangen, für den auch die Bezeichnung Genitiv verwendet wird. Die Postpositionen kön- 
nen entweder mit dem Nominativ oder mit dem Adessiv-Genitiv verbunden werden. Im Tscheremissischen steht das Bezugswort der Postposition im Genitiv, wenn es sich um ein Pronomen handelt und oft auch dann, wenn ein belebtes Wesen ausgedrückt wird, ansonsten im Nominativ. Im Mordwinischen können die Postpositionen an den Genitiv, Ablativ oder Nominativ treten. Im Ostseefinnischen und Lappischen steht das Bezugswort der Postposition meistens im Genitiv. Es kann also für äusserst wahrscheinlich gelten, dass die Postpositionen noch in der finnisch-permischen Ursprache, wenn auch vielleicht nicht ausschliesslich, so doch hauptsächlich mit dem Genitiv verbunden wurden. Wäre es nun denkbar, dass die Konstruktion Nominativ + Postposition der ugrischen Sprachen leichter zu einem Wort fusioniert wurde als die Konstruktion Genitiv + Postposition der finnisch-permischen Sprachen, wo die Endung - $n$ des Genitivs als relativ starkes Zeichen der Wortgrenze fungiert hätte? Für eine positive Antwort spricht hier, dass in den permischen Sprachen, wo die Postpositionen normalerweise an den Nominativ treten, häufiger als in den anderen finnisch-permischen Sprachen Kasus auftreten, die aus Postpositionskonstruktionen entstanden sind, sowie auch die Tatsache, dass in den permischen Sprachen, vor allem im Syrjänischen, die mit einem Nominativ beginnenden Postpositionskonstruktionen häufig als Kompositum begegnen (z.B. va-vilin 'auf dem Wasser'), die mit einem Adessiv-Genitiv beginnenden aber nicht (z.B. wotj. solen bordaz 'neben ihr') (vgl. Rédei 1962 S. 202-204). Wenn also die Postposition leichter mit einem Bezugswort im Nominativ als einem im Genitiv fusioniert werden konnte, dann ist klar, dass sich in den ugrischen Sprachen gerade eine Erweiterung des Kasussystem auf Postpositionsgrundlage anbot, während in den finnisch-permischen Sprachen ein anderer Weg zu wählen war, die Verwendung von Suffixkombinationen.

\section{Der typologische Aspekt}

Unser Thema hängt auf das engste auch mit der Sprachtypologie zusammen. Die festgestellte Entwicklungstendenz der 
Kasussysteme der finnisch-ugrischen Sprachen hat den synthetischen Charakter dieser Sprachen stark erhöht. Durch die Ikonizität, die in den auf Postpositionsbasis wie durch Suffixkombinationen erweiterten Kasussystemen zum Ausdruck kommt, wird wiederum die Agglutinativität aufrechterhalten und gestärkt. Der Grundzug einer agglutinierenden Sprache liegt darin, dass die Morpheme in bestimmter Form ohne grosse morphophonemische Veränderungen aneinander treten und dass jeder grammatischen Funktion ein Morphem und jedem Morphem eine Funktion entspricht, d.h. es begegnen keine Synonymie, Homonymie und Polysemie der grammatischen Morpheme, auch keine sog. Portmanteau-Morpheme. All diese Eigenschaften sind durch die Entwicklung des Kasussystems der fiu. Sprachen verstärkt worden.

Im Lichte der Entwicklung des Kasussystems sieht es so aus, als gehe die uralische Ursprache auf eine weniger agglutinierende Sprachform zurück. Die uralische Ursprache in der Form, wie die Rekonstruktionen sie zeigen, enthält sehr viel Homonymie der grammatischen Morpheme. Denken wir nur daran, welche verschiedenen Funktionen die Suffixe ${ }^{*} n,{ }_{j} j$ und ${ }^{*} k$ hatten. Ferner hat es eine Synonymie der Morpheme gegeben, d.h., einer Funktion entsprachen mehrere Morpheme, z.B. Pl. $*_{-} t, *_{-j}, *_{-} n$ und $*_{-} k$ sowie Lat. $*_{-} n, *_{-j}$ und $*_{-} k$. Diese Merkmale sowie der heute nur noch sehr lückenhaft dokumentierbare Vokalwechsel (z.B. fi. palaa 'brennen intr.' : polttaa 'brennen tr.', ung. hal 'sterben' : holt 'tot') können von einer voruralischen flektierenden Sprachform zeugen. Das ist übrigens auch von Robert Austerlitz (1970) angenommen worden, hauptsächlich mit sprachgeographischen Begründungen. Jedenfalls scheint der Grad der Agglutination und der Synthese vom Ururalischen an in den meisten finnisch-ugrischen Sprachen zugenommen zu haben, bis er in einigen Sprachen, vor allem im Lappischen, Estnischen und Livischen, im Laufe der beiden letzten Jahrtausende wieder eine sinkende Tendenz gehabt hat.

Die gegenseitigen Abhängigkeitsverhältnisse der zu den verschiedenen Ebenen der Grammatik gehörenden Eigenschaften im Bereich von Phonologie, Morphologie und Syntax lassen Gesetzmässigkeiten erkennen, die den Charakter von Universa- 
lien haben. Sie sind in der letzten Zeit erforscht worden u.a. von Joseph H. Greenberg (1963), Robin Lakoff (1972), Theo Vennemann (1974, 1975), Raimo Anttila (1977), Esa Itkonen (im Druck) und - vor allem unter Berücksichtigung der fiu. Sprachen - Vladimir Skalička (1968). Bekannt sind die Ausführungen Greenbergs (1963) über die in der Grundwortfolge verankerten grammatischen Eigenschaften, die er als Universalien bezeichnet. Er stellt u.a. fest, dass in Sprachen, deren Wortfolge dem Schema SOV (= Subjekt + Objekt + Verb) folgt, das Genitivattribut allgemein vor dem Bezugswort steht und dass sie als morphologische Mittel Postpositionen und Suffixe verwenden. Gerade SOV ist auch die ursprüngliche Wortstellung in den fiu. Sprachen, die auch heute noch in den meisten Sprachen dieser Wortfamilie herrscht. Wie wir gesehen haben, sind die Regeln SOV und "Genitiv vor Bezugswort" nur Sonderfälle der allgemeineren Regel "'Modifikator vor Bezugswort", und die Postpositions- und Suffixsysteme sind wenigstens in der Deklination diachron aus der Wortfolge ableitbar.

Interessant ist, dass die westlichsten fiu. Sprachen, das Ostseefinnische und Lappische, die Grundwortstellung SOV schon lange gegen die Reihenfolge SVO eingetauscht haben, vielleicht infolge germanischen Einflusses. Gerade in diesen Sprachen treten am zahlreichsten auch nichtagglutinierende Züge auf, vor allem im Lappischen, Estnischen und Livischen, die bereits eindeutige Flexionssprachen sind. Für eine flektierende Sprache charakteristische Merkmale in diesen drei Sprachen sind die Fusion von Affixen mit dem Stamm, so dass keine eindeutige Grenze auszumachen ist, der Reichtum von Lautwechseln im Stammesinnern, das Fehlen der Vokalharmonie, das Fehlen von Possessivsuffixen (Estn.), Homonymie und Synonymie der Endungen, der Reichtum an Portmanteau-Morphemen, die Verwendung von Nebensätzen, der Reichtum an Kongruenz, die relativ freie Worstellung, der Reichtum an Konjunktionen und das Fehlen des Nominalsatzes (vgl. Skalička 1968). Einige dieser Merkmale, wie die Wortfolge, die Kongruenz und das Fehlen des Nominalsatzes, können auf fremden Einfluss zurückgehen. Wieweit die anderen Faktoren 
mit diesen syntaktischen Merkmalen zusammenhängen, bleibt hier unbeantwortet.

MIKKO KORHONEN

\section{BIBLIOGRAPHIE}

Alhoniemi, Alho 1968 a. Suomen ja tšeremissin kielen suuntasijajärjestelmien funktionaalisesta rakenteesta. - Sananjalka 10.

1968 b. Über die distributive Verwendung des tscheremissischen Postposition $\gamma \hat{\partial} t \check{s}$. - Congressus secundus internationalis fenno-ugristarum. Helsinki 23. - 28. VIII. 1965. Pars I. Helsinki.

1977. Zur Verwendung des Trennungskasus im Tscheremissischen. FUF 42.

Anttila, Raimo 1972. An Introduction to Historical and Comparative Linguistics. New York.

1977. Analogy. - Trends in Linguistics. State-of-the-Art Reports. The

Hague.

Austerlitz, Robert 1970. Agglutination in Northern Eurasia in Perspective. -

Studies in General and Oriental Linguistics. Presented to Shirô Hattori on the Occasion of His Sixtieth Birthday. Tokyo.

Bárczi Géza - Benkő Loránd - Berrár Jolán 1967. A magyar nyelv története. Budapest.

Budenz József 1873-81. Magyar-ugor összehasonlító szótár. Budapest.

Collinder, Björn 1960. Comparative Grammar of the Uralic Languages. Uppsala.

Fuchs, D. R. 1927. Zur Etymologie der syrj. Postposition -miś, -miśs , -miśsti, -miśten, -miśta, -miśta, -miśtan. - FUF 18.

Greenberg, Joseph H. 1963. Some Universals of Grammar with Particular Reference to the Order of Meaningful Elements. - Universals of Language. (Ed. Joseph H. Greenberg.) Cambridge/Mass.

Hajdú Péter 1976. Bevezetés az uráli nyelvtudományba. Harmadik kiadás. Budapest.

Hakulinen, Lauri 1968. Suomen kielen rakenne ja kehitys. Kolmas, korjattu ja lisätty painos. Keuruu.

Itkonen, Erkki 1962. Die Laut- und Formenstruktur der finnisch-ugrischen Grundsprache. - UAJb 34.

1966 a. Kieli ja sen tutkimus. Helsinki.

1966 b. Esiintyykö $s$-latiivi permiläisissä kielissä? - Virittäjä.

Itkonen, Esa. (Im Druck.) Short-Term and Long-Term Teleology in Linguistic Change. - Papers from the IIIrd International Conference on Historical Linguistics. (Ed. P. Maher.)

Jakobson, Roman 1965. Quest for the Essence of Language. - Diogenes 51. Klemm Antal 1928. Magyar történeti mondattan. - A magyar nyelvtudomány kézikönyve II: 6. Budapest. 
Korhonen, Mikko 1974. Die Konjugation im Lappischen. Morphologisch-historische Untersuchung. II. Die nominalen Formkategorien. - MSFOu 155. Helsinki.

1975. Merkmalhaftigkeit und Merkmallosigkeit in den finnisch-ugrischen Lokalkasussystemen. - Congressus tertius internationalis fenno-ugristarum Tallinnae habitus 17.-23. VIII 1970. Pars I. Tallinn.

Lakoff, Robin 1972. Another Look at Drift. - Linguistic Change and Generative Theory. Essays from UCLA 1969. (Ed. by Robert P. Stockwell and Ronald K. S. Macaulay.) Bloomington/Ind.

Liimola, Matti 1963. Zur historischen Formenlehre des Wogulischen. MSFOu 127. Helsinki.

Meillet, Antoine 1921. Linguistique historique et linguistique générale. Paris.

Oinas, Felix J. 1961. The Development of some Postpositional Cases in BaltoFinnic Languages. - MSFOu 123. Helsinki.

Osnovy 1974 = Основы финно-угорского языкознания. Вопросы происхождения и развития финно-угорских языков. Москва.

Osnovy 1975 = Основы финно-угорского языкознания. Прибалтийскофинские, саамский и мордовские языки. Москва.

Paasonen, H. 1897. Kielellisiä lisiä suomalaisten sivistyshistoriaan. - Suomi III: 13 .

Peirce, Charles Sanders 1955. Philosophical Writings. (Ed. Justus Buchler.) New York.

Posti, Lauri 1937. Eräs itämerensuomalainen genetiivimuodostus. -Virittäjä.

Ravila, Paavo 1935. Die Stellung des Lappischen innerhalb der finnisch-ugrischen Sprachfamilie. - FUF 23.

1941. Über die Verwendung der Numeruszeichen in den uralischen Sprachen. - FUF 27.

Rédei (Radanovics), Károly 1962. Die Postpositionen im Syrjänischen unter Berücksichtigung des Wotjakischen. Budapest.

1968. Széljegyzetek Vászolyi Erik '’Prolatívusz, transzitívusz, lokatívusz a zürjénben, a votjákban és a finnugor alapnyelvben" c. cikkéhez. NyK 70 .

Sapir, Edward 1921. Language. New York.

Skalička, V. 1968. Über die Typologie der finnisch-ugrischen Sprachen. Congressus secundus internationalis fenno-ugristarum. Helsinki 23. - 28. VIII. 1965. Pars I. Helsinki.

SKES $1955=$ Y. H. Toivonen: Suomen kielen etymologinen sanakirja. I. Lexica Societatis Fenno-ugricae XII,1. Helsinki.

Szinnyei, Josef 1922. Finnisch-ugrische Sprachwissenschaft. Zweite, verbesserte Auflage. Leipzig.

1927. Magyar nyelvhasonlítás. Hetedik, javított és bővített kiadás. Finnugor kézikönyvek III. Budapest.

Uotila, T. E. 1933. Zur Geschichte des Konsonantismus in den permischen Sprachen. - MSFOu 65. Helsinki.

Vennemann, Theo 1974. Topics, Subjects, and Word Order: from SXV to SVX 
via TVX. - Historical Linguistics I. (Eds. Anderson, John M. and Jones, Charles.) Amsterdam.

1975. An Explanation of Drift. - Word Order and Word Order Change. (Ed. Li, Charles N.) Texas.

Wichmann, Yrjö 1924. Zur permischen Grammatik. - FUF 16.

Wichmann, Yrjö - Fuchs, D. R. 1954. Wotjakische Chrestomathie mit Glossar. Anhang: Grammatikalischer Abriss. Zweite, ergänzte Auflage. Hilfsmittel für das Studium der finnisch-ugrischen Sprachen II. Turku.

Wichmann, Yrjö - Uotila, T. E. 1942. Syrjänischer Wortschatz nebst Hauptzügen der Formenlehre. - Lexica Societatis Fenno-ugricae VII. Helsinki. 